\title{
New "knitted" EGIS esophageal stent allows atraumatic inside-out removal by inversion
}

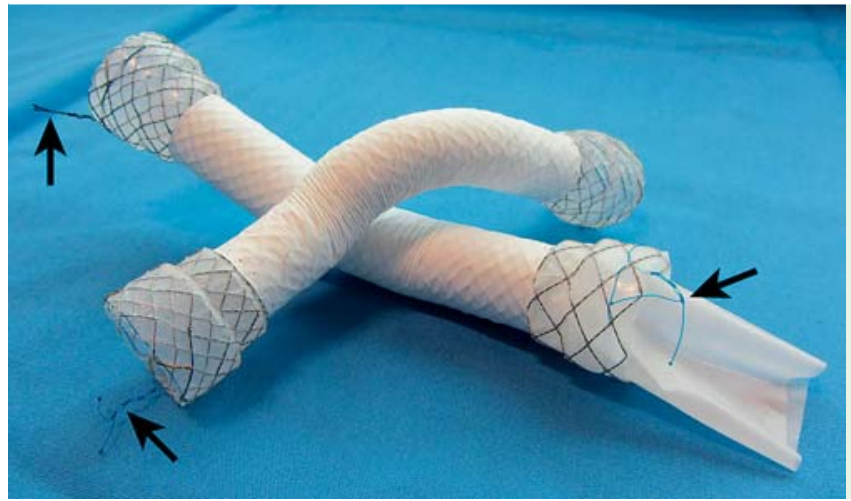

Fig. 1 Open and valved e-PTFE (woven polytetrafluoroethylene)-covered EGIS esophageal stent with proximal and distal removal strings (arrows). Note the absence of straightening forces.

Modern esophageal stents can be extracted by capture of a purse string. Under traction, braided stents lengthen and narrow. However, strictures are often friable, and extraction can cause hemorrhage or perforation.

A new nitinol stent (EGIS; S\&G Biotech, Seoul, Korea) consists of a "knitted" construction of interlinked wires, as used in enteral stents. Unlike in braided stents, no straightening forces exist within the skeleton, resulting in a resistance-free threedimensional conformation ( $\bullet$ Fig. 1 ) and reduced stent shortening on release compared with braided stents. Stent segments may move against each other, and the distal end can be inverted into the trunk by traction on the lower purse string. The stent peels away from the esophagus, reducing shearing forces on the surrounding tissues.

We present two cases of stent removal in this novel fashion.

Case 1: A 61-year-old man with a benign post-radiation stricture had suffered a perforation into the left main bronchus after balloon dilation during insertion of a biodegradable stent. The fistula was successfully occluded with an 18-mm, 9-cm EGIS esophageal stent. Bronchoscopy suggested healing, and stent removal was attempted under conscious sedation. Using rat-toothed forceps, the distal retrieval string was captured ( $\bullet$ Fig. 2 ) and the distal end of the stent inverted segment by segment, resulting in a sensation of falling dominoes. Removal was easy and atraumatic, but unfortunately the fistula remained.
Case 2: A 69-year-old woman with a dilation-resistant post-radiation stricture had a biodegradable stent inserted. This did not expand adequately, resulting in episodes of food impaction. An 18-mm, 13-cm EGIS anti-reflux stent was placed coaxially, resulting in complete expansion of the biodegradable stent. The anti-reflux stent was removed by inversion 20 days later ( Fig. 3, Video 1), leaving the fully expanded biodegradable stent in situ, with resulting grade-1 dysphagia.

Interventionists must be aware of continued developments in stents, in order to offer patients the most appropriate option.

\section{Endoscopy_UCTN_Code_TTT_1AO_2AZ}

Competing interests: Derek Edwards currently serves as International Director for S\&G Biotech. Hans-Ulrich Laasch has acted as technical consultant for S\&G Biotech.

\section{Video 1}

Fluoroscopic view of endoscopic stent removal. After performing a J-manoeuvre in the stomach, the lower removal string result is captured outside the anti-reflux valve and the distal end of the stent inverted into the stent trunk by withdrawal of the endoscope. The stent invaginates into itself, peeling away from the esophageal wall rather than pulling through the stricture. Note the radiopaque markers from the outer radiolucent biodegradable stent, which is now fully expanded.
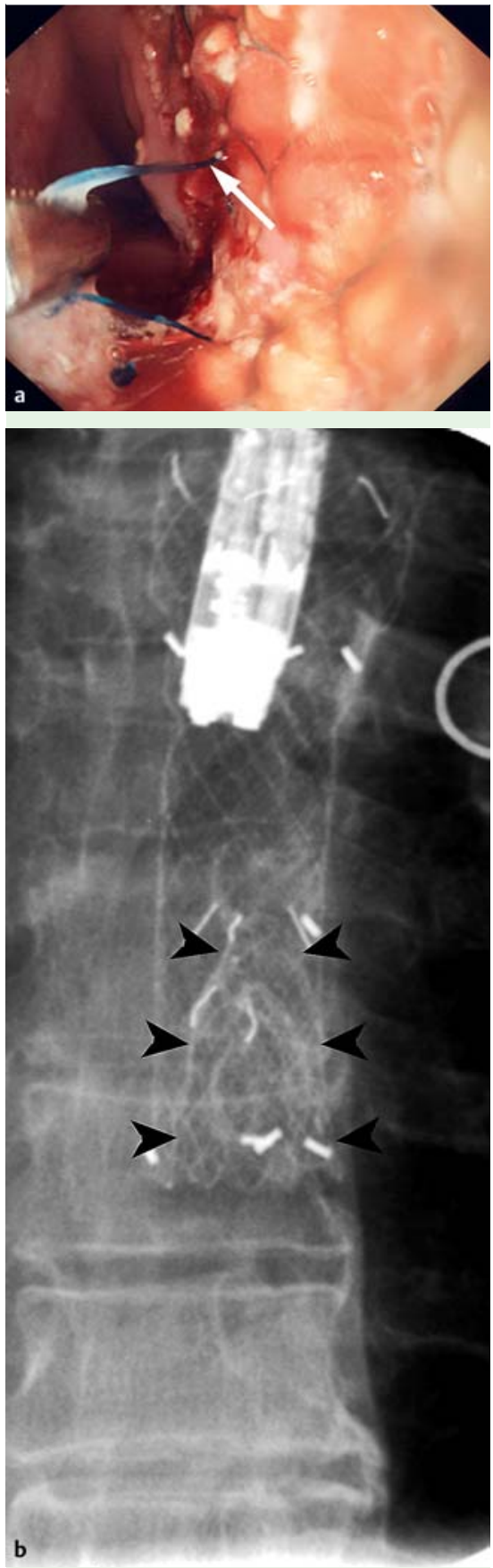

Fig. 2 a Removal of an unvalved mid-esophageal stent: capture of the lower purse string (arrow) with endoscopic forceps. b The distal end of the stent is seen as a funnel (arrowheads) inverted into the stent trunk. The stent peels off the esophageal wall rather than pulling through the stricture. Note the dense radiopaque markers from the outer radiolucent biodegradable stent. 


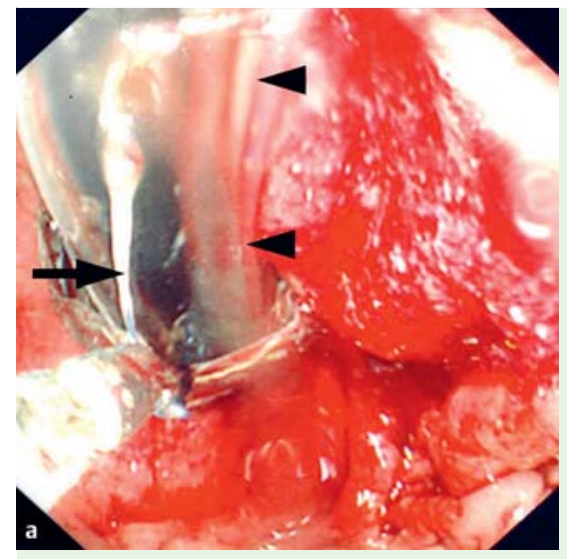

Fig.3 a Removal of an anti-reflux stent placed across the cardia: capture of the distal purse string (arrow) with the endoscope inverted in the stomach. Arrowheads: anti-reflux valve. b Extracted stent after cleaning. Note the stent is now inside-out, with the knitted skeleton outside the e-PTFE (woven polytetrafluoroethylene) membrane.

(Inset: close-up of the stent construction.)

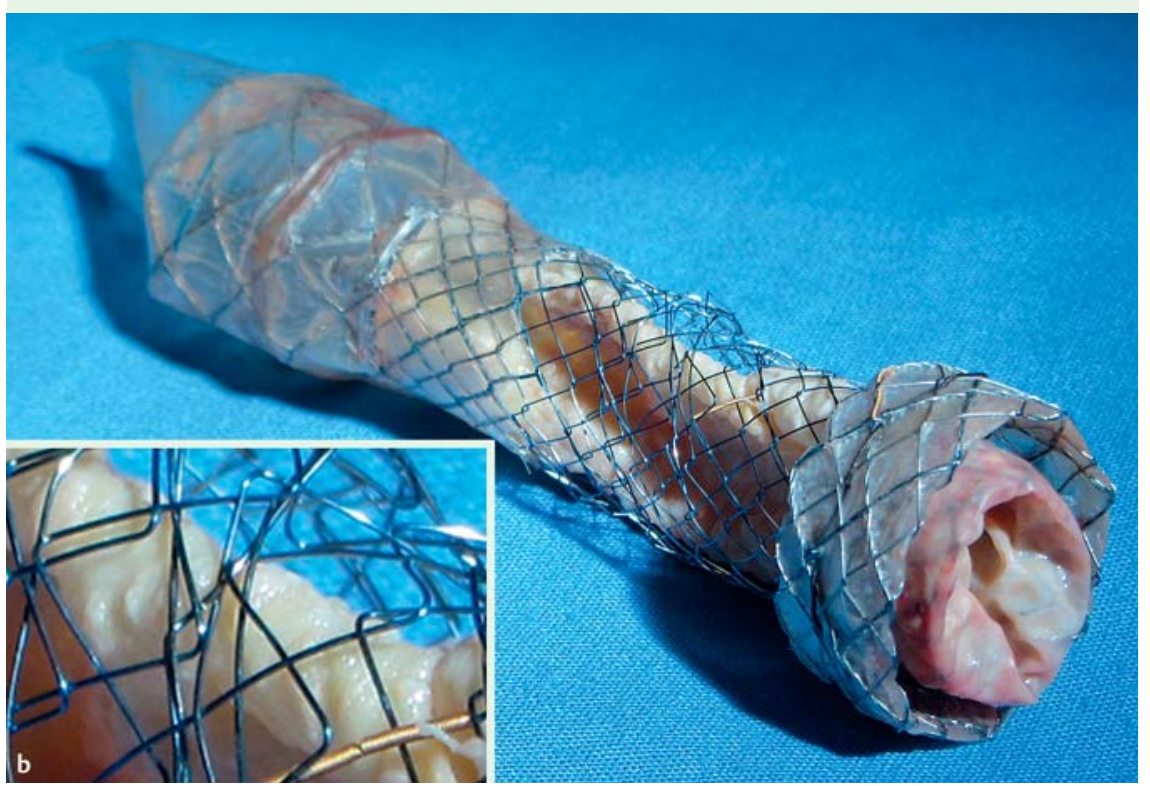

J. Suntharanathan ${ }^{1}$, D. W. Edwards ${ }^{1}$, D. Mullan ${ }^{1}$, D. F. Martin², H.-U. Laasch

${ }^{1}$ Department of Radiology, The Christie NHS Foundation Trust, Manchester, UK 2 Academic Department of GI-Radiology, South Manchester University Hospitals NHS Foundation Trust, Manchester, UK

\section{Bibliography}

DOI http://dx.doi.org/

10.1055/s-0032-1310147

Endoscopy 2013; 45: E187-E188

(c) Georg Thieme Verlag KG

Stuttgart · New York

ISSN 0013-726X

\section{Corresponding author \\ H.-U. Laasch, MD}

Department of Radiology

The Christie NHS Foundation Trust

Wilmslow Road

Manchester

M20 4BX

UK

Fax: +44-161-4468532

HUL@christie.nhs.uk 